\title{
Digital predistortion of power amplifiers using look-up table method with memory effects for LTE wireless systems
}

Ruchi Singla and Sanjay Sharma*

\begin{abstract}
Digital predistortion (DPD) techniques are widely used to linearize of RF power amplifiers. In this article, a memory polynomial-based power has been modeled with memory order of 5 and nonlinearity order of 9 . These specifications had been found suitable for advanced long-term evolution (LTE) wireless systems. A suitable DPD model was created based on the least square error minimization. Results for the complementary cumulative distribution function and normalized mean squared error were also computed. The adjacent channel leakage power ratio and error vector magnitude calculations showed that the proposed DPD for LTE system performed within the required limits as desired.
\end{abstract}

Keywords: Digital predistortion (DPD), Error vector magnitude (EVM), Look-up table (LUT), Power amplifier (PA)

\section{Introduction}

Wideband signals such as those used in long-term evolution (LTE) systems are spectrally more efficient. These signals are applied to power amplifiers (PAs), which are essential components in communication systems but are inherently nonlinear. The nonlinearity generates spectral re-growth, which leads to adjacent channel interference and violations of the out-of-band emission requirements mandated by regulatory bodies. It also causes in-band distortion, which degrades the bit error rate (BER) performance [1]. To reduce the nonlinearity, the PA can be operated at a lower power ("backed off") so that it operates within the linear portion of its operating curve. For the communications system architect and the RF PA designer, the new wireless formats introduce a number of challenges. Designers must determine the performance gap between their existing 3G designs and tomorrow's 4G operating environments, and whether these 3G designs will need to be redesigned, or a new vendor qualified [2]. The hardware must also meet or exceed absolute performance metrics such as ACPR, error vector magnitude (EVM), or throughput (e.g., BLER, BER, and PER), while also meeting internal product design

\footnotetext{
* Correspondence: skumar@rediffmail.com

Department of Electronics and Communication Engineering, Thapr University, Patiala, India
}

goals. Because smart phones and other advanced wireless devices rely so heavily on battery power, getting the most efficiency out of a design is critical. The RF PA plays a particularly key role in choosing and designing the right PA to meet design goals which is a significant challenge. Also, newer transmission formats, such as wideband code division multiple access (WCDMA) and orthogonal frequency division multiplexing (802.11ac and LTE-Advanced), have high peak-to-average power ratios (PAPR); that is, large fluctuations in their signal envelopes. This means that the PA needs to be backed off well below its maximum ("saturated") output power in order to handle infrequent peaks, which results in very low efficiencies, typically less than $10 \%$. With $>90 \%$ of the DC power being lost and turning into heat, the amplifier performance, reliability, and ongoing operating expenses are all degraded $[3,4]$.

A pre-distorter applies distortion to the input signal in order to drive the PA harder. The digital predistortion (DPD)-PA cascade attempts to combine two nonlinear systems into one linear result which allows the PA to operate closer to saturation. Beyond this point, no increase in power will suffice to linearize the PA. The PAPR of the signal greatly restricts optimal performance of the DPD system. A CDMA signal, for example, may have a PAPR as high as $13 \mathrm{~dB}$. A PA transmitting such a signal

\section{穴 Springer}

(c) 2012 Kumar and Singla; licensee Springer. This is an Open Access article distributed under the terms of the Creative Commons Attribution License (http://creativecommons.org/licenses/by/2.0), which permits unrestricted use, distribution, and reproduction in any medium, provided the original work is properly cited. 
must operate with significant back-off to prevent peaks from occurring beyond saturation. There are two common types of DPD implementation: the first is an analog implementation using a physical nonlinear device, the second and perhaps more popular choice is a digital signal processor hardware implementation where the DPD function is defined algorithmically through software [5].

There are two classes (memoryless model and model with memory) for DPD. Memoryless models focus on the PA that has a memoryless nonlinearity, i.e., the current output depends only on the current input through a nonlinear mechanism [6]. This instantaneous nonlinearity is usually characterized by the AM/AM and $\mathrm{AM} / \mathrm{PM}$ responses of the PA, where the output signal amplitude and phase deviation of the PA output are given as functions of the amplitude of its current input. Both memoryless polynomial algorithm and look-up table-based algorithm are two key algorithms for memoryless models.

As the signal bandwidth gets wider, such as in WCDMA, mobile WiMAX and 3GPP LTE and LTEadvanced (up to $100 \mathrm{MHz}$ bandwidth, five component carriers of carrier aggregation), PAs begin to exhibit memory effects. This is especially true for those high PAs used in wireless base stations. The causes of the memory effects can be attributed to thermal constants of the active devices or components in the biasing network that have frequency-dependent behaviors. As a result, the current output of the PA depends not only on the current input, but also on past input values. In other words, the PA becomes a nonlinear system with memory. For such a PA, memoryless predistortion can achieve only very limited linearization performance. Therefore, digital predistorters must need to have memory structures [7].

The most important algorithm for models with memory for DPD implementation is Volterra series and its derivatives. The most general way to introduce memory is to use the Volterra series. However, the large number of coefficients of the Volterra series makes it unattractive for practical applications. Therefore, there are several Volterra's derivatives including Wiener, Hammerstein, Wiener-Hammerstein, parallel Wiener structures and memory polynomial model are popular in digital predistorters. The so-called "memory polynomial" is interpreted as a special case of a generalized Hammerstein model and is further elaborated by combining with the Wiener model. A memory polynomial predistorter uses the diagonal kernels of the Volterra series, and is used to linearize PAs with memory effects. The predistorter is constructed using the indirect learning architecture, thereby eliminating the need for model assumption and parameter estimation of the PA $[8,9]$.

\section{Proposed model for DPD in LTE systems}

In the DPD architecture, $x(n)$ is the input signal to the predistortion unit, whose output $z(n)$ feeds the PA to produce output $y(n)$. The most general form of nonlinearity with $Q+1$ taps of memory is described by the Volterra series, which consists of a sum of multidimensional convolutions. In the training branch, the Volterra series pre-distorter can be described by

$$
z(n)=\sum_{k=1}^{K} Z_{k}(n)
$$

where

$$
z_{k}(n)=\sum_{m_{1}=0}^{Q} \ldots \sum_{m_{1}=0}^{Q} h_{k}\left(m_{1}, \ldots, m_{k}\right) \prod_{l=1}^{k} y\left(n-m_{1}\right)
$$

is the $k$-dimensional convolution of the input with Volterra kernel $h_{k}$. This is a generalization of a power series representation with a finite memory of length $Q+1$. The $z(n)$ also can be written as follows

$$
\begin{aligned}
z(n)= & h_{0}+\sum_{m_{1}=0}^{Q} h_{1}\left(m_{1}\right) y\left(n-m_{1}\right) \\
& +\sum_{m_{1}=0}^{Q} \sum_{m_{2}=0}^{Q} h_{2}\left(m_{1}, m_{2}\right) y\left(n-m_{1}\right) y\left(n-m_{2}\right)+\ldots
\end{aligned}
$$

The DPD is described by a memory polynomial

$$
z(n)=\sum_{k=1}^{K} \sum_{q=0}^{Q} a_{k q} x(n-q)|x(n-q)|^{k-1}
$$

where the $x(n)$ and $z(n)$ are complex input signal and output signal of DPD model, respectively. The polynomial includes both the odd and even order terms. $K$ and $Q$ are the highest nonlinear and memory orders. $a_{k q}$ are the complex coefficients of the polynomial that is to be extracted in this model. The memory polynomial can be described by the topology below (Figure 1).

The coefficients of the polynomial are extracted by indirect learning structure indicated in Figure 2.

The algorithm uses two identical memory polynomial models for the predistorter and training. The real predistorter block is the exact copy of the training block. It has $x$ $(n)$ as input and $z(n)$ as output. In the training branch, the memory polynomial predistorter can be described by

$$
z(n)=\sum_{k=1}^{K} \sum_{q=0}^{Q} a_{k q} y(n-q)|y(n-q)|^{k-1}
$$

where $y(n)$ and $z(n)$ are the input and output of the predistorter in the training branch, respectively, and $a_{k q}$ are the coefficients of the predistorter. If $Q=0$, the structure in the equation degenerates into a memoryless polynomial. 

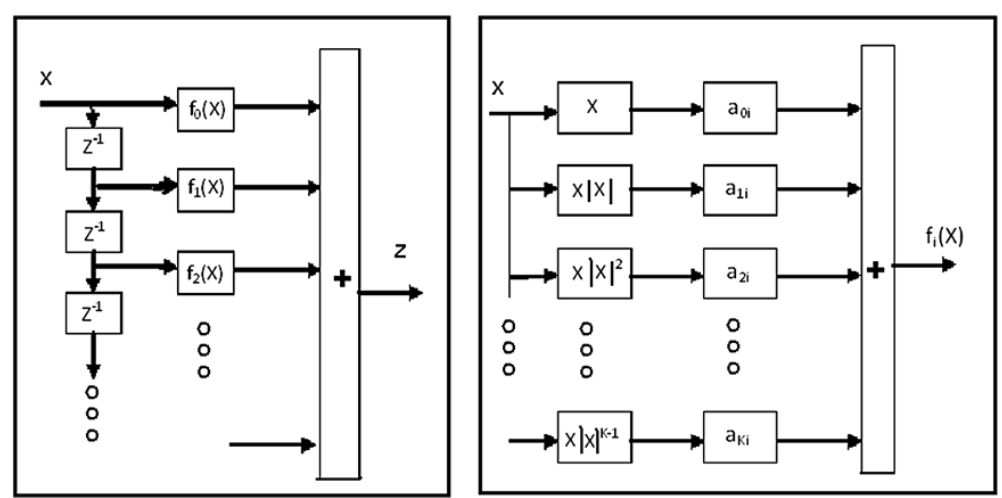

Figure 1 Structure of the memory polynomial.

Since the model is linear with respect to its coefficients, the predistorter coefficients $a_{k q}$ can directly be obtained using a least-squares algorithm by defining a new sequence:

$$
u_{k q}(n)=\frac{y(n-q)}{G}\left|\frac{y(n-q)}{G}\right|^{k-1}
$$

At convergence, we have

$$
\begin{aligned}
& z=\left[z(0), z(1), \ldots, z(N-1)^{T}\right] \\
& u=\left[u_{10}, \ldots, u_{K 0}, \ldots, u_{1 Q}, \ldots, u_{K Q}\right] \\
& u_{k q}=\left[u_{k q}(0), u_{k q}(1), \ldots, u_{k q}(N-1)^{T}\right] \\
& a=\left[a_{10}, \ldots, a_{k 0}, \ldots, a_{1 Q}, \ldots, a_{K Q}\right]^{T}
\end{aligned}
$$

The least squares solution is given by

$$
\hat{a}=\left(U^{H} U\right)^{-1} U^{H} z
$$

where $(U)^{H}$ denotes the complex conjugate transpose matrix.

After getting memory polynomial coefficients $a=\left[a_{10}, \ldots\right.$, $\left.a_{k 0}, \ldots, a_{1 Q}, \ldots, a_{K Q}\right]^{T}$ and loading these coefficients into a nonlinear filter (Figure 3), the memory polynomial predistorter is able to function properly [10]. Figure 4 describes the block diagram of the overall DPD system.

The model identification methods in the design are the least squares error using QR decomposition and least squares error using singular value decomposition. In the model identification, the PA output is normalized by the small-signal gain and then used as the input of the DPD model [11]. The PA input is taken as the output of the DPD model. Only part of the signal is used to do model identification. Accurate synchronization of the PA input and output signal is implemented in this algorithm [12-14]. The PA input and output after normalization and delay adjustment are given as output of this model. Normalized mean squared error (NMSE) is used to evaluate how close the PA input and the DPD model output. Highest nonlinear order $K$ and memory order $Q$ are the key parameters that decide how well the DPD model works. To meet the requirements of the NMSE, $K$ and $Q$ values were increased step-by-step. Finally, the values of $K$ and $Q$ chosen were 5 and 7, respectively. These values were required to justify the memory effects of RF PA deployed in wideband communication systems especially LTE systems. Those samples with amplitude less than threshold would not be used in model extraction. No sample was

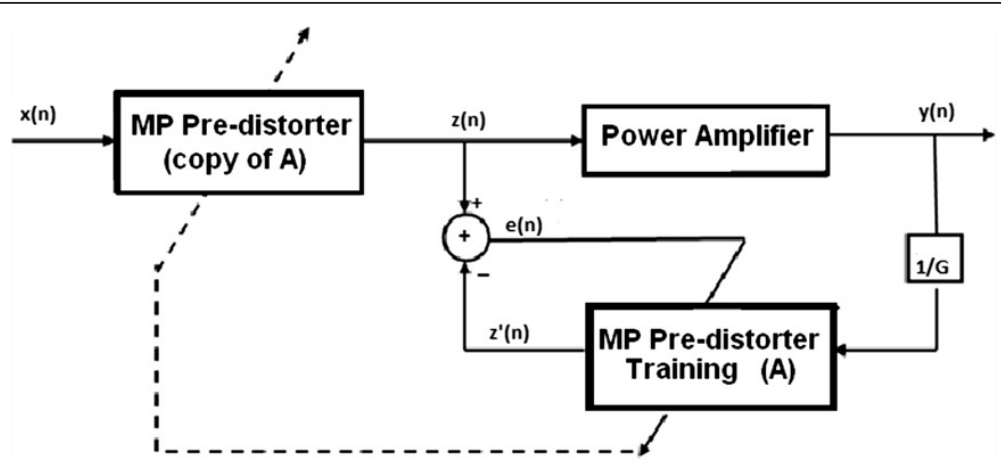

Figure 2 Indirect learning structure to extract the coefficients of the polynomial. 


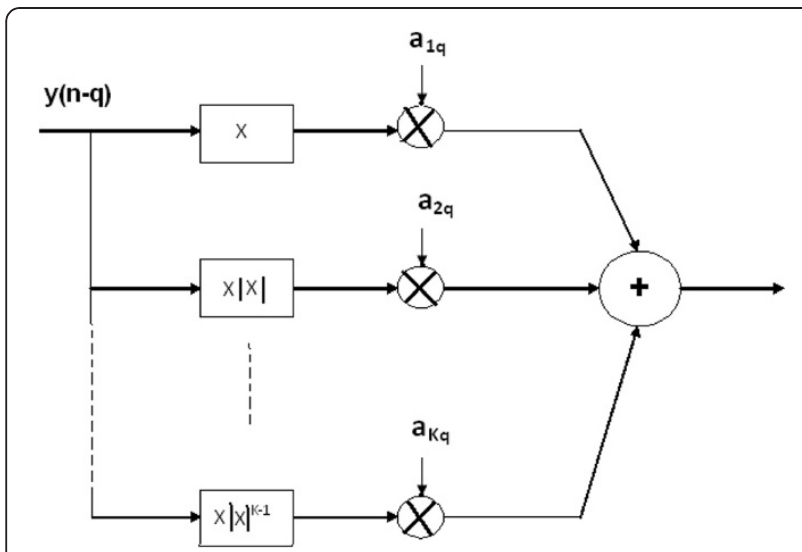

Figure 3 Memory polynomial structure describing the behavior of nonlinear PA for coefficient extraction.

discarded because the threshold level used was 0 . This model is used to extract the memory polynomial model that describes the behavior of nonlinear PA. The PA output is normalized by the small signal gain and shifted to accurately synchronize with the PA input. The result is given as shifted PA output. After the normalization, the small-signal gain is $1(0 \mathrm{~dB})$. Behavior models of PA were traditionally developed for narrowband applications. The models are extracted based on the AM/AM and AM/PM characteristics without considering memory effects. A complex polynomial of instantaneous input power is used to approximate the PA gain. However, with the increase of signal bandwidth, memory effects cannot be ignored anymore. The AM/AM and AM/PM characteristics are not constant, but change as the function of both the present and the past input signals. Several models were proposed to describe the PA memory effects [15].
The average input power should be kept to the level that the peak output power of the PA does not exceed 3$\mathrm{dB}$ gain compression point. Otherwise, the extraction error will be large. The system was designed for an LTE bandwidth of $20 \mathrm{MHz}$, base DFT size of 2048, and transmission bandwidth configuration, expressed in units of resource blocks is chosen as 100 . The total power dynamic range is the difference between the maximum and the minimum transmit power of an OFDM symbol for a specified reference condition and is set to $20 \mathrm{~dB}$, the cyclic prefix used is normal and the modulation scheme used is 64-QAM.

The EVM is a measure of the difference between the ideal symbols and the measured symbols after the equalization. This difference is called the error vector. The value of EVM recommended for LTE systems for $64-\mathrm{QAM}$ is $8 \%$. Adjacent channel leakage power ratio (ACLR) is the ratio of the filtered mean power centered on the assigned channel frequency to the filtered mean power centered on an adjacent channel frequency and the minimum requirement of ACLR in LTE systems is set to $45 \mathrm{~dB}$.

The basic unit of EVM measurement is defined over one sub-frame $(1 \mathrm{~ms})$ in the time domain and subcarriers $(180 \mathrm{kHz})$ in the frequency domain:

$$
E V M=\sqrt{\frac{\sum_{t \in T} \sum_{f \in F(t)}\left|Z^{\prime}(t, f)-I(t, f)\right|^{2}}{\sum_{t \in T} \sum_{f \in F(t)}\left|I(t, f)^{2}\right|}}
$$

where $T$ is the set of symbols with the considered modulation scheme being active within the sub frame, $F(t)$ is the set of subcarriers within the subcarriers with the considered modulation scheme being active in symbol $t, I(t, f)$ is the ideal signal reconstructed by the

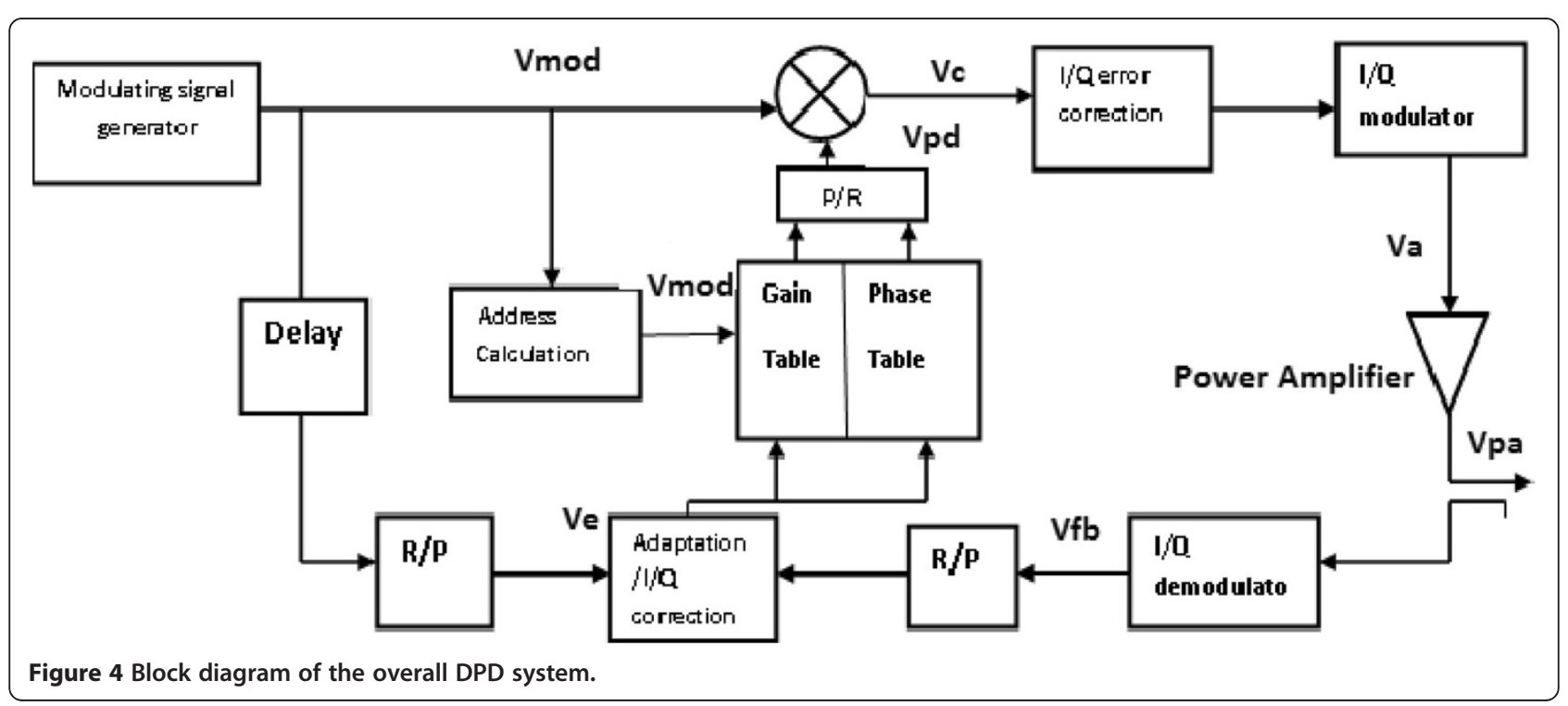




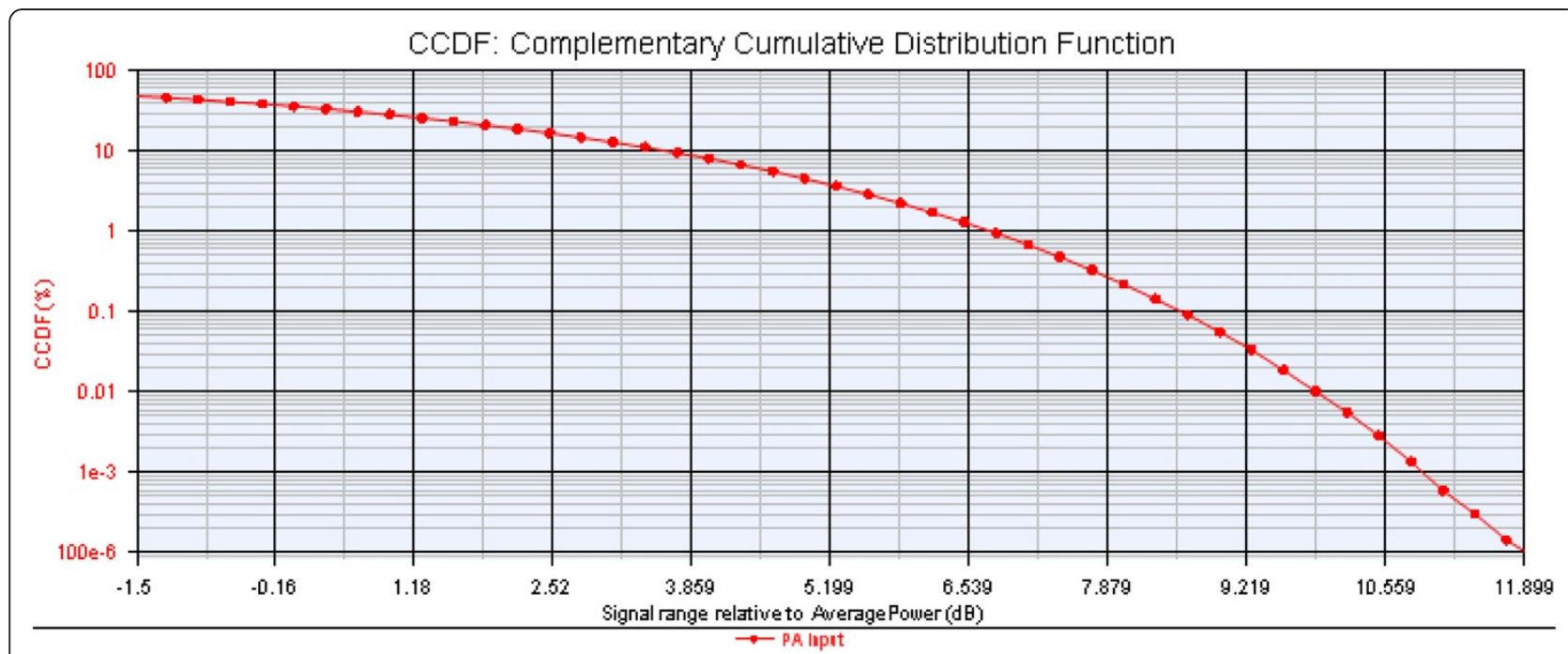

Figure 5 Graph for the complimentary cumulative distribution function.

measurement equipment in accordance with relevant Tx models, $Z^{\prime}(t, f)$ is the modified signal.

Implicit in the definition of EVM is an assumption that the receiver is able to compensate a number of transmitter impairments. The signal under test is equalized and decoded according to

$$
Z^{\prime}(t, f)=\frac{F F T\left\{z(v-\Delta \tilde{t}), e^{-j 2 \Pi \Delta \tilde{f} v}\right\} e^{-j 2 \Pi f \Delta \tilde{t}}}{\tilde{a}(t, f), e^{j \tilde{\phi}(t, f)}}
$$

where $z(v)$ is the time domain samples of the signal under test, $\tilde{t}$ is the sample timing difference between the FFT processing window in relation to nominal timing of the ideal signal, $\tilde{f}$ is the RF frequency offset, $\tilde{\phi}(t, f)$ is the phase response of the Tx chain and $\tilde{a}(t, f)$ is the amplitude response of the Tx chain. The NMSE value is expressed in $\mathrm{dB}$

$$
N M S E[d B]=10 * \log _{10}\left[\frac{\sum\left|\mathbf{x}_{\mathbf{i}}-\mathbf{y}_{\mathbf{i}}\right|^{2}}{\sum\left|\mathbf{x}_{\mathbf{i}}\right|^{2}}\right]
$$

The DPD module predistorts the signal in a reverse manner. The input of the DPD model is the normalized output of the PA, and the desired output of the extracted DPD model is the input of the PA. For DPD model NMSE evaluation, $x_{i}$ is measured PA input signal, $y_{i}$ is the extracted DPD model output signal. For PA model NMSE evaluation, $x_{i}$ is measured PA output

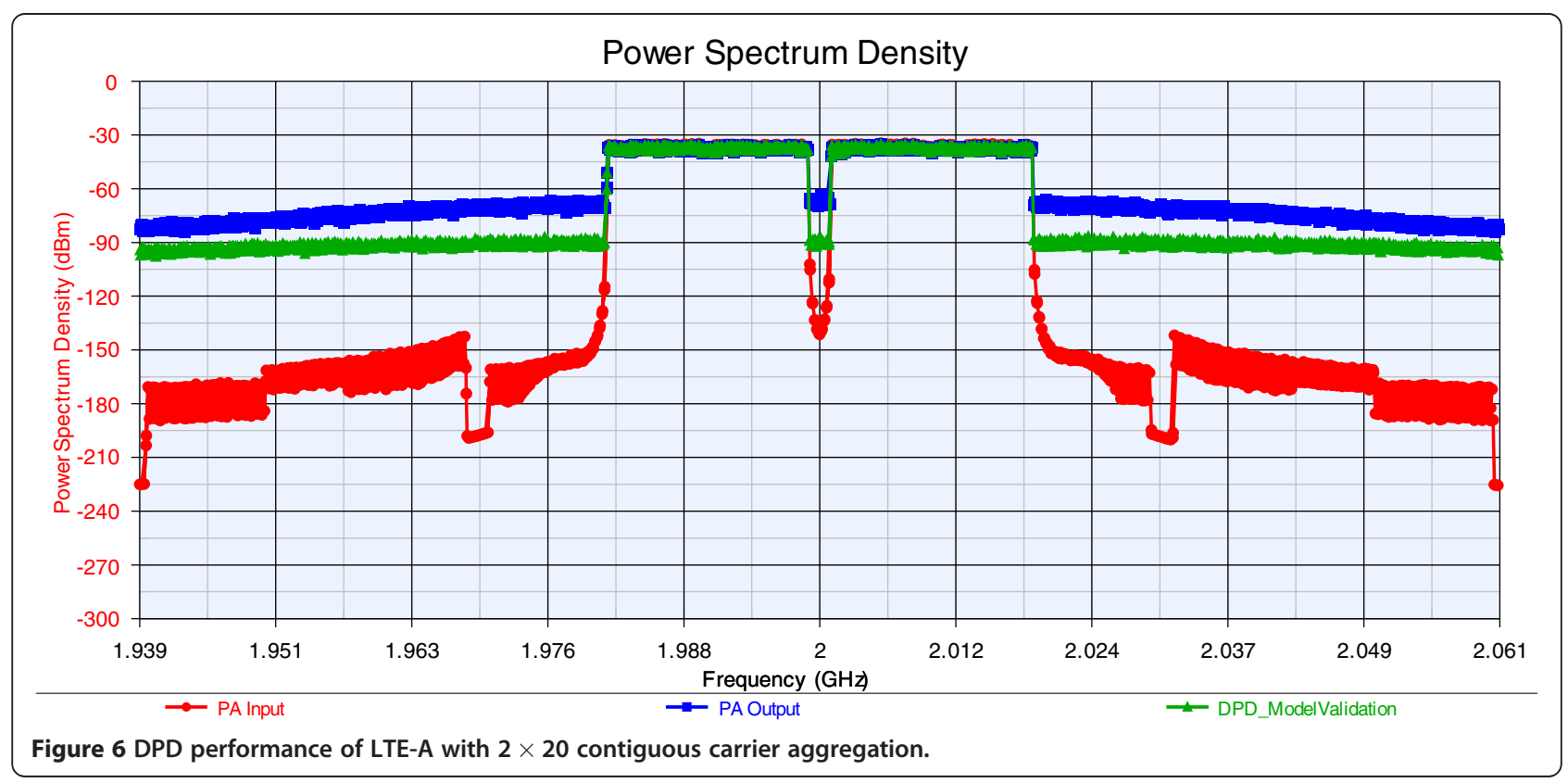




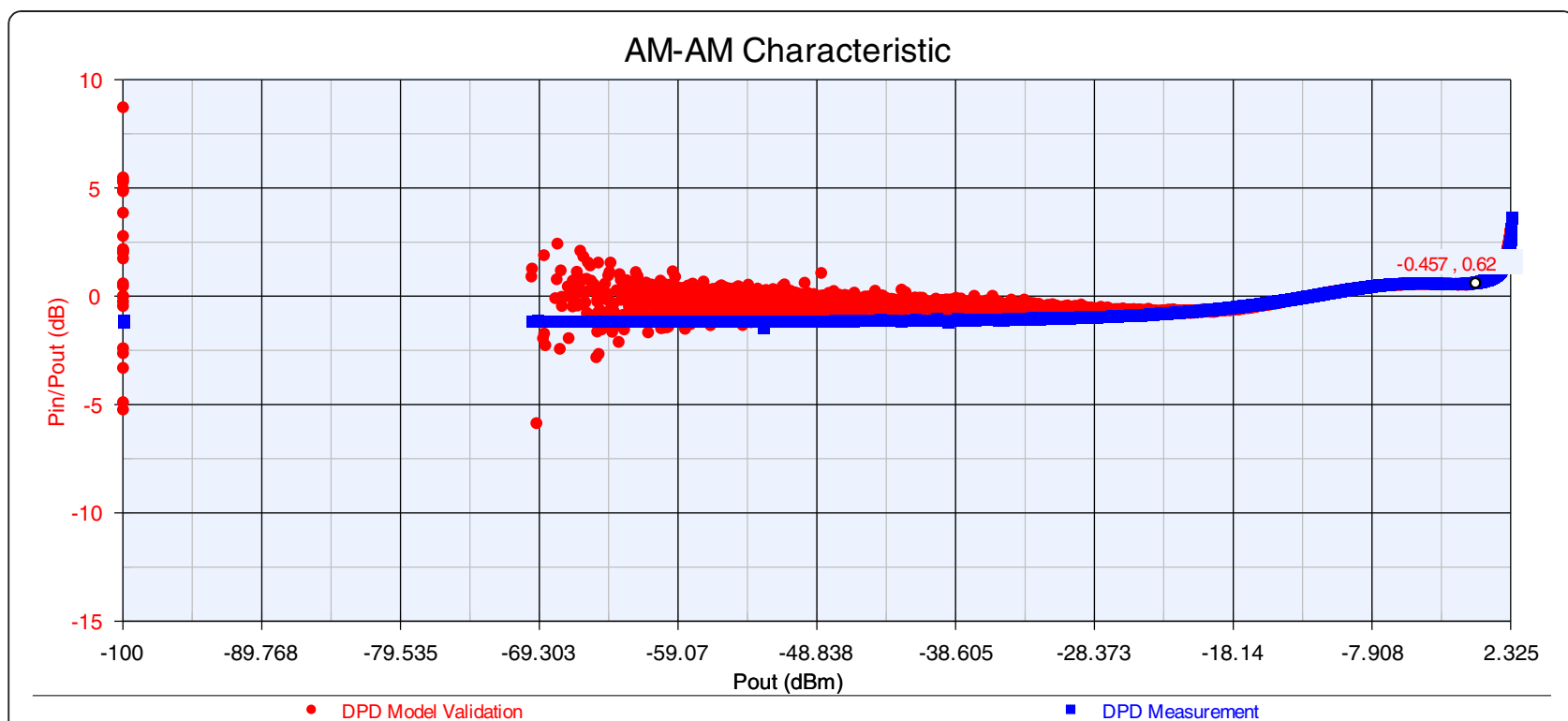

Figure 7 AM-AM characteristics.

signal, $y_{i}$ is the extracted PA model output signal. The smaller the NMSE, the more accurate the model. The recommended NMSE is less than $-35 \mathrm{~dB}$. If the NMSE is too large, the extracted model cannot work effectively or even fails. Increasing the nonlinear order or memory order may improve NMSE. If the NMSE is still not low enough after nonlinear/memory order adjustment, lowering the operating point of PA is suggested.

The PA model is used to adjust the value of the baseband signal and the input signal of the extracted DPD model to the same range. The PA output normalized by small-signal gain is taken as the input of DPD model.
When the extracted DPD model is implemented, the actual input signal is the baseband signal. To make the DPD model work correctly, the baseband signal should be adjusted to the same range as the normalized output of the PA. The gain is the adjustment factor. Typically, the gain adjustment is implemented between the signal source and digital predistorter model as shown below. The value of gain is the adjustment factor calculated in this model.

\section{Results and discussion}

Engineers migrating to $4 \mathrm{G}$ require a solution that makes implementing DPD fast and practical for 4G communications

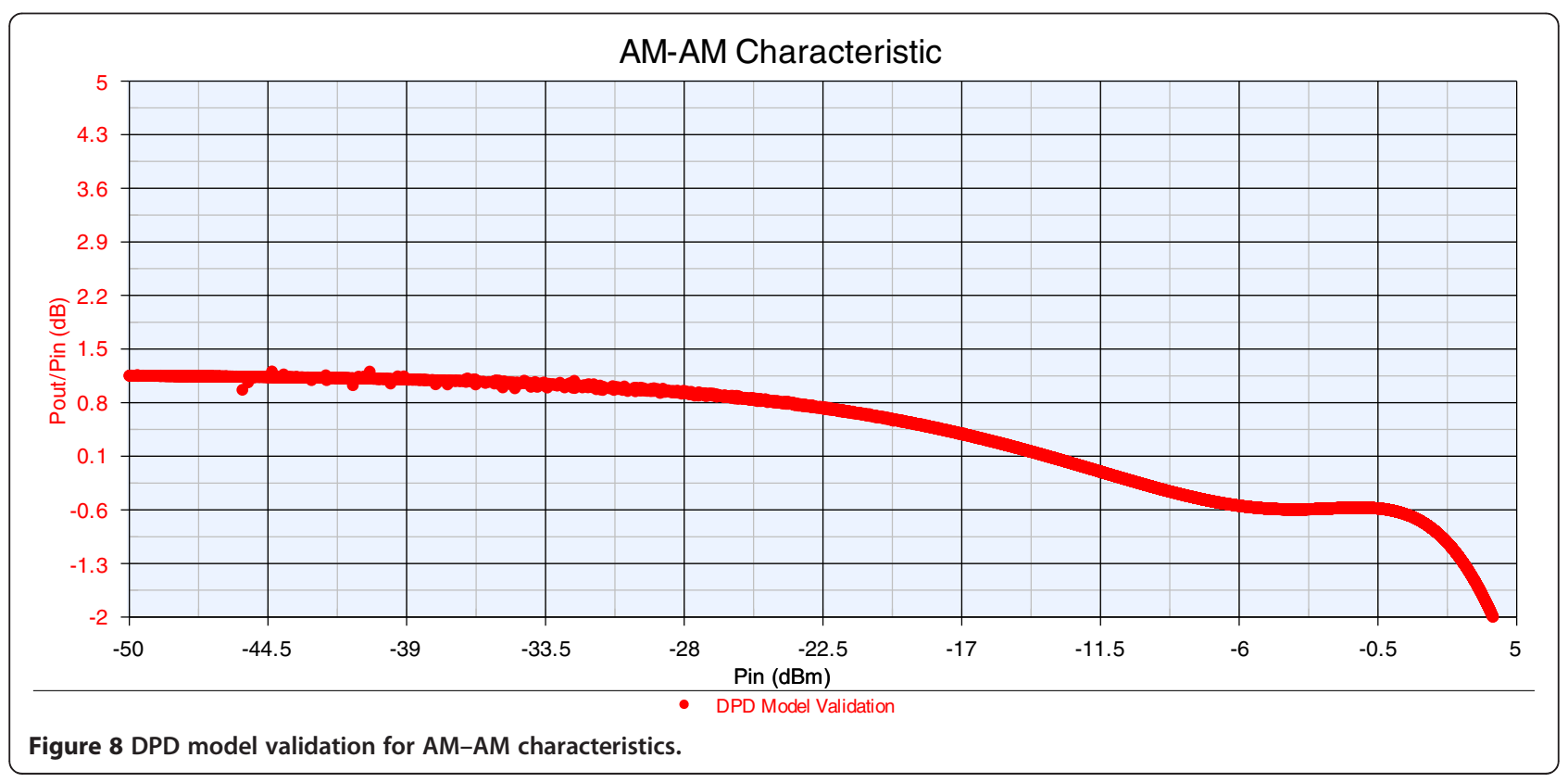




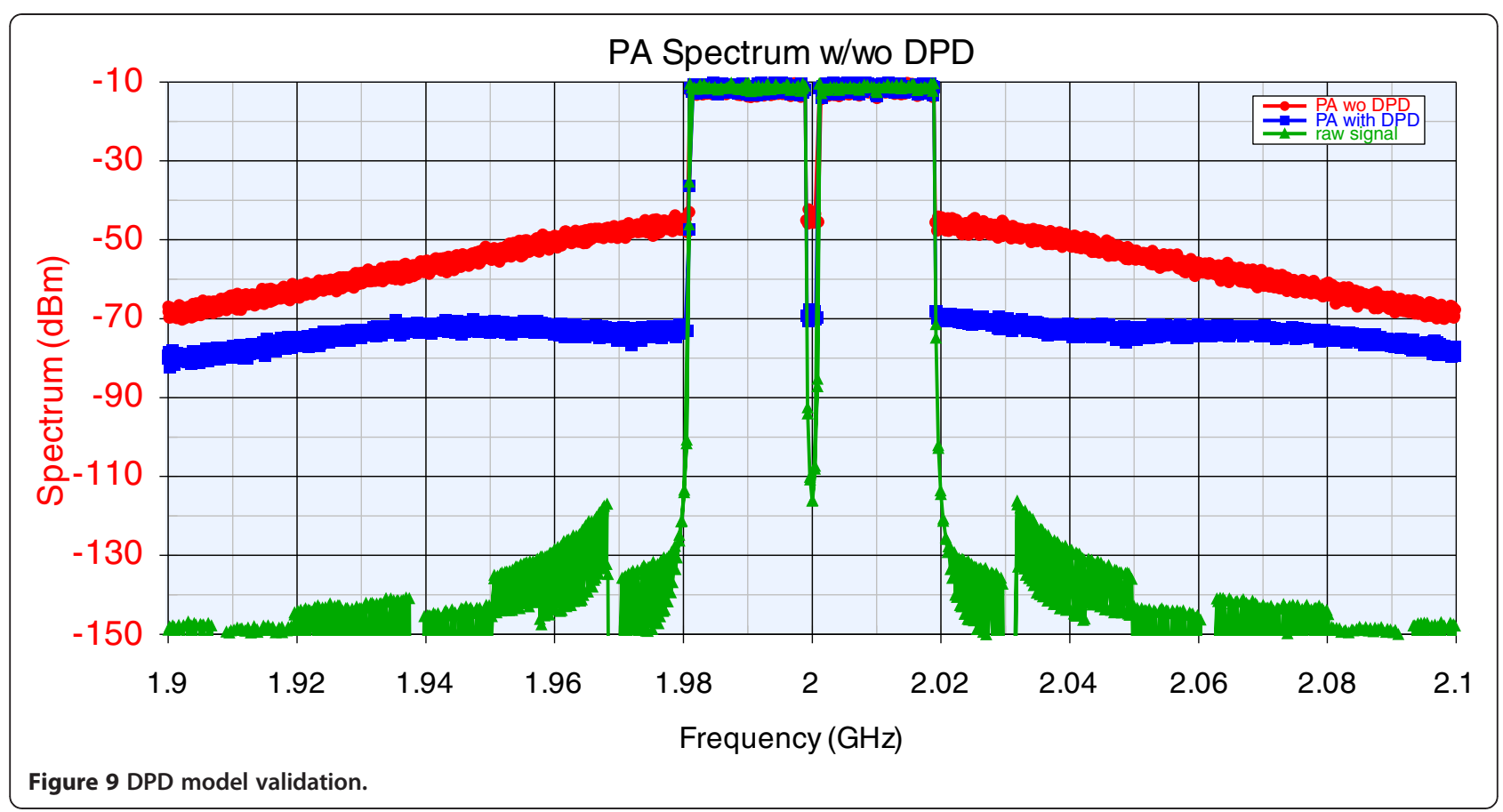

systems, i.e., one that can be used by engineers at all levels of expertise and requires minimal equipment. In modern communication systems, spectrally efficient wideband RF signals have a PAPR as high as $13 \mathrm{~dB}$. CFR preconditions the signal to reduce signal peaks without significant signal distortion. By reducing PAPR, CFR allows the PA to operate more efficiently at higher power levels, without impacting compliance with spectral mask and EVM specifications. CFR acts on the signal itself, whereas DPD corrects for the PA nonlinearity, allowing the signal to be run even higher.

Complementary cumulative distribution function (CCDF) measurements provide important information for engineers involved in the design and manufacturing of system components used in fourth generation (4G) networks. 4G networks use OFDM that results in higher quality voice as well as greater data rates for cellular services. The peak-to-average ratio of $4 \mathrm{G}$ components is dependent on the subchannels used on a particular channel within a spread spectrum signal format. This can impact the distortion of a transmitted 4G signal. Performing CCDF measurements on $4 \mathrm{G}$ systems provides power characteristics of amplified, filtered, and mixed spread spectrum signals. The CCDF measurements performed in our case are shown in Figure 5 and it provides information on the amount of time the signal spends at or above a specific power level-with the power level being in $\mathrm{dB}$ relative to the average power level. The $Y$-axis is the percent of time the signal power is at or above the power specified by the $X$-axis. The $X$ axis represents $d B$ above the average power level; this displays the peak-to-average ratios as opposed to absolute power levels. For example when $t=1 \%$ on the $Y$ axis, the corresponding peak-to-average ratio is $6.90 \mathrm{~dB}$. This means the signal power exceeds the average by at least $6.90 \mathrm{~dB}$ for $1 \%$ (correct use) of the time. The peak and mean powers obtained are 4.724 and $-3.919 \mathrm{dBm}$, which gives rise to peak-to-average power ratio of 8.643 $\mathrm{dBm}$ and is calculated as PAPR $=$ Peak power - Mean power.

Figure 6 shows an LTE-advanced signal with $2 \times 20$ $\mathrm{MHz}$ carrier aggregation $(40 \mathrm{MHz}$ total signal bandwidth). The PA output spectrum is shown with and without DPD. Note the use of oversampling for an actual measurement bandwidth that is wider than the original signal. Without this additional bandwidth to

Table 1 ACLR measurements

\begin{tabular}{lllll}
\hline & ACLR & & & \\
\cline { 2 - 5 } & L_2BW (dB) & L_BW (dB) & U_2BW (dB) & U_BW (dB) \\
\hline Original ACLR & 64.69 & 48.93 & 47.859 & 61.334 \\
ACLR after PA & 63.462 & 44.812 & 44.473 & 60.751 \\
ACLR after PA + DPD & 64.462 & 48.783 & 47.769 & 61.474 \\
\hline
\end{tabular}


quantify the out-of-band energy (distortion products), it is not possible to correct for this spectral regrowth later.

The PA input is displayed in green, the distorted PA output without DPD is displayed in blue, and the linearized DPD + PA output is shown in red.

Figure 7 shows the DPD AM-AM characteristics terms of two curves. The red curve is the AM-AM of the samples that are used to do model extraction, and the blue curve is the AM-AM of the samples that are not used for model extraction. Figure 8 gives the DPD model validation as far as the AM-AM characteristics are concerned.

The power alignment value is obtained as 0.652 and that value calculates back-off power. The NMSE value of $-48.64 \mathrm{~dB}$ is computed as it is necessary to first look at the NMSE value and then the DPD spectrum. Figure 9 provides the power spectrum before DPD and after DPD. The EVM measurements show that when the original value before PA was -26.805 , it was -24.924 after the PA stage when the DPD was not employed. And when the DPD is employed, the value of EVM was obtained as -26.801 which shows that by employing the DPD for LTE systems, the errors are minimized to a great extent.

Table 1 shows the ACLR measurements at three stages which involve the stage before PA, after the PA, and then finally after the PA and after the DPD was employed. In LTE systems, the minimum requirement of ACLR has been set to $45 \mathrm{~dB}$. Table 1 clearly shows the deterioration in the ACLR values after the PA stage and when DPD is employed, the ACLR values crosses beyond the threshold limits.

\section{Conclusion}

As engineers migrate to the advanced wireless communication systems such as LTE-advanced or 802.11ac, choosing and designing the right PA to meet design goals at the lowest possible cost becomes more difficult, both for base stations and mobile devices. Because DPD enables the PA to be operated with high efficiency, near saturation, and without significant signal distortion, it allows engineers to address many base stations/mobile device PA design challenges. This article considered the design of DPD systems to linearize PAs with memory effects. By adding a digital predistorter in the baseband, the PA is allowed to operate into its nonlinear region, thereby significantly increasing its efficiency. The efficiency gain translates into electricity and cooling cost savings for service providers and longer battery life for mobile terminal users. The challenge here is to address the memory effects exhibited by the higher PAs or the PAs for wideband signals. In addition, analog components in the transmitter have imperfections that need to be compensated as well.

\section{Competing interests}

The authors declare that they have no competing interests.

Received: 3 March 2012 Accepted: 2 October 2012

Published: 31 October 2012

\section{References}

1. JS Kenney, A Leke, Wireless report: power amplifier spectral regrowth for digital cellular and PIIS applications. Microwave J. 3, 74-92 (1995)

2. D Lei, GT Zhou, DR Morgan, M Zhengxiang, JS Kenney, K Jaehyeong, CR Giardina, A robust digital baseband predistorter constructed using memory polynomials. IEEE Trans. Commun. 52(1), 159-165 (2004)

3. SP Stapleton, FC Costescu, An adaptive predistorter for a power amplifier based on adjacent channel emissions. IEEE Trans. Veh. Technol. 41(1), 49-56 (1992)

4. Raytheon Systems Co, LP Strickland, CP Yates, J Patel, FG Muir, LH Goree, CJ Briden, CE Phase III final Review (, 2001)

5. PB Kenington, DW Bennett, Linear distortion correction using a feed forward system. IEEE Trans. Veh. Technol. 45(1), 474-480 (1996)

6. R Singla, SK Sharma, Low complexity look up table based adaptive digital predistorter with low memory requirements. EURASIP J. Wirel. Commun. Netw. 2012, 43 (2012). doi:10.1186/1687-1499-2012-43

7. D Lei, Digital predistortion of power amplifiers for wireless applications (Georgia Institute of Technology, USA, 2004). PhD thesis

8. S Roland, Adaptive power amplifier linearization by digital pre-distortion with narrowband feedback using genetic algorithms (Georgia Institute of Technology, USA, 2005). PhD thesis

9. M Helaoui, S Boumaiza, A Ghazel, FM Ghannouchi, Power and efficiency enhancement of $3 \mathrm{G}$ multicarrier amplifiers using digital signal processing with experimental validation. Part 1. IEEE Trans. Microwave Theory Tech. 54 (4), 1396-1404 (2006)

10. HA Suraweera, KR Panta, M Feramez, J Armstrong, OFDM peak-to-average power reduction scheme with spectral masking, in IEEE Proceedings of Symposium on Communication Systems, Networks and Digital Signal Processing, 2004, pp. 164-167

11. C Zhao, J Baxley Robert, ZG Tong, D Boppana, KJ Stevenson, Constrained clipping for crest factor reduction in multiple-user OFDM, in IEEE proceedings of Radio and Wireless Symposium, 2007, pp. 341-344

12. V Olli, Digital modulators with crest factor reduction techniques (Helsinki University of Technology, Finland, 2006). PhD thesis

13. S Boumaiza et al., On the RF/DSP design for efficiency of OFDM transmitters. IEEE Trans. Microwave Theory Tech. 53(7), 2355-2361 (2005)

14. S Boumaiza, Advanced memory polynomial linearization techniques, in IEEE International Microwave Symposiu (Boston, 2009)

15. Amplifier pre-distortion linearization and modeling using $x$-parameters available at www.home.agilent.com/agilent/redirector.jspx

doi:10.1186/1687-1499-2012-330

Cite this article as: Singla and Sharma: Digital predistortion of power amplifiers using look-up table method with memory effects for LTE wireless systems. EURASIP Journal on Wireless Communications and Networking 2012 2012:330.

\section{Submit your manuscript to a SpringerOpen ${ }^{\circ}$ journal and benefit from:}

- Convenient online submission

- Rigorous peer review

- Immediate publication on acceptance

- Open access: articles freely available online

- High visibility within the field

- Retaining the copyright to your article

Submit your next manuscript at $>$ springeropen.com 